\title{
Reasons for the failure of B.Tech Students in Mathematics using Combined Disjoint Blocked Fuzzy Cognitive Maps (CDBFCM)
}

\author{
G.Anusha
}

\begin{abstract}
At the present time most of the B.Tech. students are failing in Mathematics subject. The reason is they are not having fundamentals. Lack of practice also one reason. Because most B.Tech. Students are with attitude problem. In this paper we are going to investigate the causes for the failure of B.Tech Students in Mathematics with the help of Combined Disjoint Block Fuzzy Cognitive Maps (CDBFCM). W.B. Vasantha Kandasamy, A. Victor Devadoss started the technique. This technique will be efficient if the numeral of concepts are big in figure and we have to cluster them. The troubles are going to be discussed here with the assist of Combined Disjoint Block Fuzzy Cognitive Maps (CDBFCM). Finally, we are going to identify the most important causes for the failure of B.Tech. students in Mathematics. For this we used neutrosophic device. There are five sections. Section one provides details regarding Fuzzy Cognitive Maps and the the causes for the failure of B.Tech. students in Mathematics. Section two provides basic concepts of Fuzzy Cognitive Maps, Combined Disjoint Block Fuzzy Cognitive Maps. Process of finding the unseen outline was given in section three. The difficulties are given in section four. After the completion of work decisions are given in the last section.

Keywords: Combined Disjoint Blocked Fuzzy Cognitive Maps, Failure of Mathematics, B.Tech. students
\end{abstract}

\section{INTRODUCTION}

FCM look like neural networks, they contain commanding, extensive things like a numerical instrument to model multifaceted models. A mathematical model which is started through L.A. Zadeh in 1965 named as Fuzzy Cognitive Maps. After ten years in 1976, Political scientist R. Axelord [7] used these cognitive maps for making decision in communal and political systems. Bart Kosko [2],[3],[4] was the guru of fuzzy logic, and he introduced this idea in the year 1986. Also he enhanced the power of cognitive maps considering fuzzy principles to concepts of the cognitive maps, fuzzy degrees of interconnection among concepts. He utilizes this for indicating data like a consistent, heading for, bi- level-logic diagram On this concept number of research papers presented by using FCM as an device on students. In this paper we will define Fuzzy Cognitive Maps

Revised Manuscript Received on December 30, 2019.

* Correspondence Author

G. Anusha*, Assistant Professor, Department of Mathematics,

Vignans Lara Institute of technology and sciences, Vadlamudi, Guntur (Dt), India.

(c) The Authors. Published by Blue Eyes Intelligence Engineering and Sciences Publication (BEIESP). This is an open access article under the CC BY-NC-ND license (http://creativecommons.org/licenses/by-nc-nd/4.0/)
(FCMs) and also define many of its related definitions. They are useful when the information is unconfirmed. The process will be very easy, efficient because using this method the data will be analyzed using directed graphs, relation matrices. There are many causes for the failure of B.tech. Students in Mathematics. In this paper, various causes for the failure of B.tech. Students in Mathematics were considered, at last the foremost reasons were found.

\section{PRELIMINARIES}

Fuzzy Cognitive Maps (FCMs) will be useful applicable if the information is unconfirmed one. The FCMs based on the estimation of experts. FCMs sculpt the earth as a set of classes and informal associations among classes.

\subsection{Definition}

If nodes for FCM be fuzzy sets subsequently these nodes will be named like fuzzy nodes

\subsection{Definition}

FCMs having end weights otherwise causal relations of set $\{-1,0,1\}$ will be named as simple FCMs.

\subsection{Definition}

An FCM is a directed graph by means of concepts similar to principles, actions and so on being nodes, casual relations being edges. This indicates fundamental connection among concepts.

\subsection{Definition}

Take nodes $D_{1}, D_{2, \ldots,} D_{n}$ belonging to the FCM. Assume that the directed graph be drawn by means of end weight $w_{i j} \in\{0,1,-1\}$. The matrix $\mathrm{W}$ is determined with $\mathrm{W}$ $=\left(w_{i j}\right)$ where $w_{i j}$ be the weight belonging to the directed edge $D_{i} D_{j}$. W will be named as adjacency matrix belonging to FCM, which is moreover called relation matrix belonging to FCM.

The most significant point is each and every matrix connected by means of FCM is constantly a square matrix having diagonal elements be 0 .

\subsection{Definition}

Let $D_{1}, D_{2}, \ldots, D_{n}$ be the nodes of an FCM. G = $\left(g_{1}, g_{2}, \ldots, g_{n}\right)$ such that $g_{i} \in\{0,1\}$. $G$ will be named as instantaneous state vector, $G$ indicates on-off situation for a node during that moment.

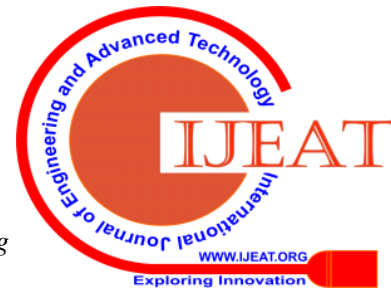




\section{Reasons for the failure of B.Tech Students in Mathematics using Combined Disjoint Blocked Fuzzy Cognitive Maps (CDBFCM)}

$g_{i}=0$ when $g_{i}$ be off, $g_{i}=1$ when $g_{i}$ be on for $\mathrm{i}=1,2$, $\ldots, \mathrm{n}$.

\subsection{Definition}

Let $D_{1}, D_{2}, \ldots, D_{n}$ be present as nodes to the FCM. Let $\overline{D_{1} D_{2}}, \overline{D_{2} D_{3}}, \ldots \overline{D_{i} D_{j}}$ exist as ends for FCM ( $\mathrm{i} \neq \mathrm{j}$ ).At that moment these ends construct a directed cycle.

An FCM will suppose to be cyclic, when that contains a directed cycle. An FCM will suppose to be acyclic when that will not have at all directed cycle.

\subsection{Definition}

If an FCM contains cycles then there will be a feedback for that FCM.

\subsection{Definition}

If an FCM contains feedback that is if the causality stream all the way by means of a cycle using innovatory method, such FCM will be named as a dynamical system.

\subsection{Definition}

Suppose $\overline{D_{1} D_{2}}, \overline{D_{2} D_{3}}, \ldots \overline{D_{n-1} D_{n}}$ exist as cycle. If $D_{i}$ be switched on, and the causal relation streams all the way by means of ends of a cycle, when it once more gives $D_{\bar{i}}$, then the dynamical system will go in a circular manner. We can apply the above situation to every node $D_{i}$ for

$\mathrm{i}=1,2, \ldots, \mathrm{n}$. The stability stage of the dynamical system will be named as hidden pattern.

\subsection{Definition}

When the stability stage for the dynamical system be an exclusive state vector, it will be named as a fixed point. Take FCM having $\left\{D_{1}, D_{2}, \ldots, D_{n}\right\}$ being nodes. In favor of illustration we will begin this dynamical system by means of making $D_{1}$ on. We will imagine such that FCM settles having $D_{1}$ and $D_{n}$ on, that is when the state vector ruins the same as $(1,0,0, \ldots 0)$ which will be named as fixed point.

\subsection{1 . Definition}

When FCM settles having state vector replicating as $G_{1} \rightarrow G_{2} \rightarrow \ldots \rightarrow G_{i} \rightarrow G_{1}$ subsequently the stability stage will be named as limit cycle.

\subsection{Definition}

Limited figure belonging to FCM will join collectively for creating combined consequence to each and every FCM. Let us take $W_{1}, W_{2}, \ldots, W_{p}$ as adjacency matrices belonging to FCM having nodes $D_{1}, D_{2}, \ldots, D_{n}$. At that time combined FCM will be obtained through totaling each and every adjacency matrix $W_{1}, W_{2}, \ldots, W_{p}$. The combined FCM adjacency matrix will be indicated as $\mathrm{W}=W_{1}+W_{2}+\ldots+W_{p}$.

\subsection{Definition}

Let us take $D_{1}, D_{2}, \ldots, D_{n}$ as $n$ different attributes for the trouble having $\mathrm{n}$ extremely big, not a prime. When $\mathrm{n}$ is separated as $\mathrm{k}$ equivalent classes, that is $\mathrm{n} / \mathrm{k}=\mathrm{t}$ and these $\mathrm{k}$ classes will be put out of joint. When the directed graph for all the $\mathrm{k}$ classes was discovered having $\mathrm{t}$ attributes in each and every class, at that time the subsequent relation matrices will be produced. All the relation matrices will be combined like blocks for getting $n \times n$ matrix. The $n \times n$ relation matrix generates combined disjoint block FCM having un equivalent classes.

\subsection{Definition}

Assume that $\mathrm{G}=\left(g_{1}, g_{s} ., g_{n}\right)$ be the vector accepted inside the dynamical system $\mathrm{W}$. Then

$\mathrm{GW}=\left(g_{1}, g_{2}^{g}, \ldots g_{n}^{\sigma}\right)$. If $\left(h_{1}, h_{2}, \ldots, h_{n}\right)$ will be obtained by thresholding, restricting the vector $\mathrm{GW}$, then it will be indicated as $\left.\left(g_{1}, g_{2}^{\prime}, \ldots g_{n}\right)\right\urcorner\left(h_{1}, h_{2}, \ldots, h_{n}\right)$.

Therefore the sign' $\urcorner$ ' indicates that resulting vector was threshold and restructured

FCMs contain lot of benefits. At the same time they have drawbacks too. Most important benifit be FCM is easy. FCMs will work using the view of a person who is familiar in that area. If information be unconfirmed at that time FCM will turn out to be useful. With the help of this method hidden pattern related to the condition will be discovered. As all of us be familiar that the power belonging to the information at all times depends on so many views we apply combined FCMs having the views of lot of persons who are familiar in this area. The drawback regarding combined FCM be if the weightages will be $1,-1$ to similar $D_{i} D_{j}$, we contain the total summing to 0 . Due to the above reason the relation matrices $W_{1}, W_{2}, \ldots W_{k}$ cannot be applicable to summation always. Joint contradictory views may lead to withdraw, using the strong law of large numbers, an agreement appears because model view accurate fundamental people view. The above trouble will be simply clarified when FCM elements be 1 and 0 .

\section{METHOD OF DETERMINING THE HIDDEN PATTERN}

Suppose $D_{1}, D_{2}, \ldots, D_{n}$ being nodes for FCM having feedback. Suppose $\mathrm{W}$ is the connected adjacency matrix. We will determine the hidden pattern if $D_{1}$ be switched on. If we give entry as the vector $G_{1}=$ $(1,0, \ldots, 0)$, information must bypass all the way through the connection matrix $\mathrm{W}$. We can perform this by means of multiplying $G_{1}$ with the matrix W. Suppose $G_{1} \mathrm{~W}=\left(g_{1}, g, \ldots, g_{n}\right)$ having threshold function, it means

replicate $g_{\mathrm{i}}$ with 1 when $g_{\mathrm{i}} \geq \mathrm{k}, g_{\mathrm{i}}$ with 0 when $g_{\mathrm{t}}<$ $\mathrm{k}$ ( $\mathrm{k}$ being appropriate positive integer).

We reconstruct the resultant concept, the concept $D_{1}$ be incorporated into the reconstructed vector by means of taking the first coordinate be 1 inside resultant vector. 
If $G_{1} \mathrm{~W} \mathrm{~W}$, replicate the $G_{2}$ at this moment take $\left.G_{2}\right\rceil$ similar process. The process be replicated until fixed point point will be obtained.

\section{CONCEPTS OF PROBLEM}

With the help from philological questionnaire, by taking the view of a person who is familiar in this area the below 15 attributes $\left\{G_{1}, G_{2}, \ldots, G_{14}, G_{15}\right\}$ are considered.

$G_{1}: \quad$ Lack of fundamentals

$G_{2}$ : Leaving some concepts as choice

$G_{3}:$ By hearting Mathematics

$G_{4}:$ Lack of practice

$G_{5}:$ Lack of application skills

$G_{6}:$ Fear towards the subject

$G_{7}:$ Taking the course by force

$G_{\mathrm{g}}$ : Method of teaching

$G_{9}$ : Attitude towards learning the subject

$G_{10}$ : Lack of connection between the subject and students

$G_{11}$ : Self doubt

$G_{12}$ : Low I.Q.

$G_{13}$ : Short attention span

$G_{14}$ : Lack of understanding about signs and symbols

$G_{15}:$ Teacher student ratio

We divide the 15 attributes into 3 classes $D_{1}, D_{2}, D_{3}$ containing 5 in each class.

$$
\text { Let } \begin{aligned}
D_{1} & =\left\{G_{1}, G_{2}, G_{10}, G_{11}, G_{14}\right\} \\
D_{2} & =\left\{G_{4}, G_{5}, G_{8}, G_{12}, G_{13}\right\} \\
D_{3} & =\left\{G_{3}, G_{6}, G_{7}, G_{9}, G_{15}\right\}
\end{aligned}
$$

We will consider the view of a person who is expert in this area to all classes, acquire the matrix connected to combined disjoint block FCMs. View of person who is familiar in this area to the class $D_{1}=\left\{G_{1}, G_{2}, G_{4}, G_{5}, G_{8}\right\}$ be in the shape of the directed graph.

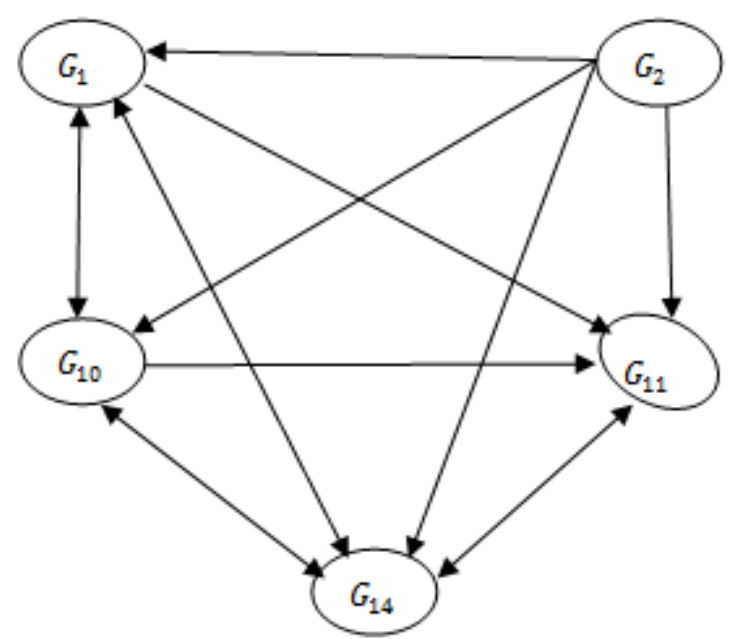

Fig.1

As per view of a person who is familiar in this area the attribute Lack of fundamentals is interconnected with Lack of connection between the subject and students, Lack of understanding about signs and symbols. The attribute Lack of fundamentals is the cause for Self doubt. The attribute leaving some concepts as choice is the cause for Lack of fundamentals, Lack of connection between the subject and students, Self doubt, Lack of understanding about signs and symbols. The attribute Lack of connection between the subject and students is the cause for Self doubt. The attributes Lack of connection between the subject and students, Lack of understanding about signs and symbols are interconnected. The attributes self doubt, Lack of understanding about signs and symbols are interconnected.

The associated relation matrix $M_{1}$ is as follows.

$M_{1}=\left[\begin{array}{lllll}0 & 0 & 1 & 1 & 1 \\ 1 & 0 & 1 & 1 & 1 \\ 1 & 0 & 0 & 1 & 1 \\ 0 & 0 & 0 & 0 & 1 \\ 1 & 0 & 1 & 1 & 0\end{array}\right]$

The view of a person who is familiar in this area to $D_{2}=$ $\left\{G_{4}, G_{5}, G_{8}, G_{12}, G_{13}\right\}$ will be in the shape of the directed graph.

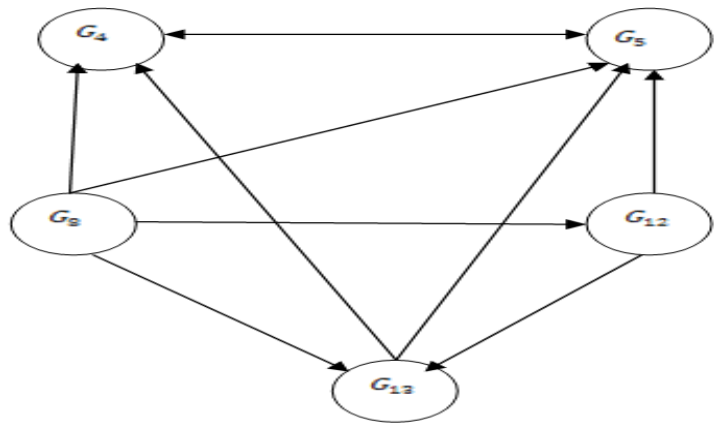

Fig.2

As per view of a person who is familiar in this area the attributes lack of practice and lack of application skills are inter connected. The attribute method of teaching is the cause for lack of practice, lack of application skills, low I.Q., short attention span. 


\section{Reasons for the failure of B.Tech Students in Mathematics using Combined Disjoint Blocked Fuzzy Cognitive Maps (CDBFCM)}

The attribute low I.Q. is the cause for lack of application skills, short attention span. The attribute short attention span is the cause for lack of practice and lack of application skills. The associated relation matrix $M_{2}$ is as follows.

$$
M_{2}=\left[\begin{array}{lllll}
0 & 1 & 0 & 0 & 0 \\
1 & 0 & 0 & 0 & 0 \\
1 & 1 & 0 & 1 & 1 \\
0 & 1 & 0 & 0 & 1 \\
1 & 1 & 0 & 0 & 0
\end{array}\right]
$$

The view of a person who is familiar in this area for the class $D_{3}=\left\{G_{3}, G_{6}, G_{7}, G_{9}, G_{15}\right\}$ will be in the shape of the directed graph.
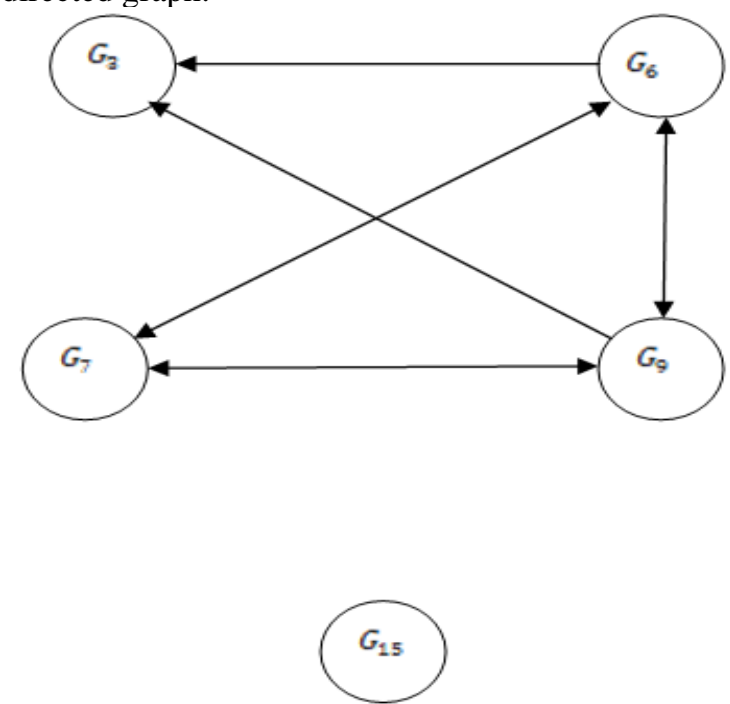

Fig.3

As per the view of a person who is familiar in this area the attribute fear towards the subject is the cause for by hearting Mathematics. The attributes fear towards the subject and taking the course by force are interconnected. The attributes fear towards the subject and Attitude towards learning the subject are interconnected. The attributes taking the course by force and attitude towards learning the subject are interconnected.

The associated relation matrix $M_{3}$ is as follows.

$$
M_{3}=\left[\begin{array}{lllll}
0 & 0 & 0 & 0 & 0 \\
1 & 0 & 1 & 1 & 0 \\
0 & 1 & 0 & 1 & 0 \\
1 & 1 & 1 & 0 & 0 \\
0 & 0 & 0 & 0 & 0
\end{array}\right]
$$

At this moment the combined disjoint block connection matrix belonging to the fuzzy cognitive maps $\mathrm{F}$ as follows.
$\mathrm{F}=$
$\left[\begin{array}{lllllllllllllll}0 & 0 & 0 & 0 & 0 & 0 & 0 & 0 & 0 & 1 & 1 & 0 & 0 & 1 & 0 \\ 1 & 0 & 0 & 0 & 0 & 0 & 0 & 0 & 0 & 1 & 1 & 0 & 0 & 1 & 0 \\ 0 & 0 & 0 & 0 & 0 & 0 & 0 & 0 & 0 & 0 & 0 & 0 & 0 & 0 & 0 \\ 0 & 0 & 0 & 0 & 1 & 0 & 0 & 0 & 0 & 0 & 0 & 0 & 0 & 0 & 0 \\ 0 & 0 & 0 & 1 & 0 & 0 & 0 & 0 & 0 & 0 & 0 & 0 & 0 & 0 & 0 \\ 0 & 0 & 1 & 0 & 0 & 0 & 1 & 0 & 1 & 0 & 0 & 0 & 0 & 0 & 0 \\ 0 & 0 & 0 & 0 & 0 & 1 & 0 & 0 & 1 & 0 & 0 & 0 & 0 & 0 & 0 \\ 0 & 0 & 0 & 1 & 1 & 0 & 0 & 0 & 0 & 0 & 0 & 1 & 1 & 0 & 0 \\ 0 & 0 & 1 & 0 & 0 & 1 & 1 & 0 & 0 & 0 & 0 & 0 & 0 & 0 & 0 \\ 1 & 0 & 0 & 0 & 0 & 0 & 0 & 0 & 0 & 0 & 1 & 0 & 0 & 1 & 0 \\ 0 & 0 & 0 & 0 & 0 & 0 & 0 & 0 & 0 & 0 & 0 & 0 & 0 & 1 & 0 \\ 0 & 0 & 0 & 0 & 1 & 0 & 0 & 0 & 0 & 0 & 0 & 0 & 1 & 0 & 0 \\ 0 & 0 & 0 & 1 & 1 & 0 & 0 & 0 & 0 & 0 & 0 & 0 & 0 & 0 & 0 \\ 1 & 0 & 0 & 0 & 0 & 0 & 0 & 0 & 0 & 1 & 1 & 0 & 0 & 0 & 0 \\ 0 & 0 & 0 & 0 & 0 & 0 & 0 & 0 & 0 & 0 & 0 & 0 & 0 & 0 & 0\end{array}\right]$

If the on position of the attribute lack of fundamentals was taken, each and every other states are off the effect of $\mathrm{Y}=$

$\left(\begin{array}{lllllllllllllll}1 & 0 & 0 & 0 & 0 & 0 & 0 & 0 & 0 & 0 & 0 & 0 & 0 & 0 & 0\end{array}\right)$ on the CDBFCM is as follows.

YF

7

$\left(\begin{array}{lllllllllllllll}0 & 0 & 0 & 0 & 0 & 0 & 0 & 0 & 0 & 1 & 1 & 0 & 0 & 1 & 0\end{array}\right)$ (Say) $Y_{1}=$

$Y_{1} \mathrm{~F}$

$\left(\begin{array}{lllllllllllllll}1 & 0 & 0 & 0 & 0 & 0 & 0 & 0 & 0 & 1 & 1 & 0 & 0 & 1 & 0\end{array}\right)$ (Say) $Y_{2}=$

$Y_{2} \mathrm{~F}$

$\left(\begin{array}{lllllllllllllll}1 & 0 & 0 & 0 & 0 & 0 & 0 & 0 & 0 & 1 & 1 & 0 & 0 & 1 & 0\end{array}\right)$ ${ }_{2}=\mathrm{Y} Y_{3}=$

$\mathrm{Y}_{2}$ will be the fixed point belonging to the dynamical system.

If the state $G_{1}$ is on lack of fundamentals, lack of connection between the subject and students, self doubt and lack of understanding about signs and symbols are causes for failure.

If the on state of the attributes lack of fundamentals, lack of practice, taking the course by force, self doubt, remaining nodes are in off state.

At this moment we learn the effect of the dynamical system $\mathrm{F}$

Let $\mathrm{T}$

$\left(\begin{array}{lllllllllllllll}1 & 0 & 0 & 1 & 0 & 0 & 1 & 0 & 0 & 0 & 1 & 0 & 0 & 0 & 0\end{array}\right)$

be the state vector depicting the on state vector $\mathrm{T}$ inside dynamical system $\mathrm{F}$.

$\mathrm{TF}$

$\left(\begin{array}{lllllllllllllll}0 & 0 & 0 & 0 & 1 & 1 & 0 & 0 & 1 & 1 & 1 & 0 & 0 & 1 & 0\end{array}\right)$ (Say) $T_{1}=$

$T_{1} \mathrm{~F}$

7

$\left(\begin{array}{lllllllllllllll}1 & 0 & 1 & 1 & 0 & 1 & 1 & 0 & 1 & 1 & 1 & 0 & 0 & 1 & 0\end{array}\right)$ $T_{2}\left(\right.$ Say) $T_{2}=\mathrm{F}_{T}$ 


$\left(\begin{array}{lllllllllllllll}1 & 0 & 1 & 0 & 0 & 1 & 1 & 0 & 1 & 1 & 1 & 0 & 0 & 1 & 0\end{array}\right)$
$=T_{3}$ (Say)
$T_{3} \mathrm{~F}$
$\left.7 \begin{array}{lllllllllllllll}1 & & & & & & & & & & & & & & \\ 1 & 0 & 1 & 0 & 0 & 1 & 1 & 0 & 1 & 1 & 1 & 0 & 0 & 1 & 0\end{array}\right)$
$T_{3}=T_{4}=$

At that time $T_{3}$ will be the fixed point of the dynamical system. Therefore factors $G_{1}, G_{4}, G_{7}, G_{11}$ will be on, factors leaving some concepts as choice, by hearting Mathematics, lack of application skills, fear towards the subject, method of teaching, attitude towards learning the subject, lack of connection between the subject and students, low I.Q., short attention span, lack of understanding about signs and symbols, teacher student ratio are in the off state all other states turn out to be on.

\section{RESULT AND DISCUSSION PART}

Here the technique applied is Combined Disjoint Blocked Fuzzy Cognitive Maps. Using this technique we will identify major causes for various real world problems. This is suitable simple, commanding device, useful in many areas like communal , medicinal. There are so many causes for the failure of B.Tech. Students in Mathematics. In this paper, various causes for the failure of B.Tech. Students in Mathematics were considered, at last important causes were found. With the help of the philological questionnaire, by taking the view of a person who is familiar in this area we have considered 15 attributes $\left\{G_{1}, G_{2}, \ldots, G_{14}, G_{15}\right\}$ which are explained above.

We divided the 15 attributes into 3 classes

$D_{1}, D_{2}, D_{3}$ each consisting of 5 attributes. They are

$$
\begin{aligned}
& D_{1}=\left\{G_{1}, G_{2}, G_{10}, G_{11}, G_{14}\right\} \\
& D_{2}=\left\{G_{4}, G_{5}, G_{8}, G_{12}, G_{13}\right\} \\
& D_{3}=\left\{G_{3}, G_{6}, G_{7}, G_{9}, G_{15}\right\}
\end{aligned}
$$

In Fig. 1 using the attributes $G_{1}, G_{2}, G_{10}, G_{11}, G_{14}$ we have drawn a directed graph. We draw the graph based on the relation between the attributes $G_{1}, G_{2}, G_{10}, \mathrm{G}_{11}, G_{14}$ which is explained below.

The attribute Lack of fundamentals is interconnected with Lack of connection between the subject and students, Lack of understanding about signs and symbols. The attribute Lack of fundamentals is the cause for Self doubt. The attribute leaving some concepts as choice is the cause for Lack of fundamentals, Lack of connection between the subject and students, Self doubt, Lack of understanding about signs and symbols. The attribute Lack of connection between the subject and students is the cause for Self doubt. The attributes Lack of connection between the subject and students, Lack of understanding about signs and symbols are interconnected. The attributes self doubt, Lack of understanding about signs and symbols are interconnected.

Using this Fig.1 and using the relations a related connection matrix $M_{1}$ is formed which is given below.

$$
M_{1}=\left[\begin{array}{lllll}
0 & 0 & 1 & 1 & 1 \\
1 & 0 & 1 & 1 & 1 \\
1 & 0 & 0 & 1 & 1 \\
0 & 0 & 0 & 0 & 1 \\
1 & 0 & 1 & 1 & 0
\end{array}\right]
$$

If there is relation between attributes we have given 1.Otherwise we have given 0 .

In Fig.2 using the attributes $G_{4}, G_{5}, G_{8}, G_{12}, G_{13}$ we have drawn a directed graph. We draw the graph based on the relation between the attributes $G_{4}, G_{5}, G_{8}, G_{12}, G_{13}$ which is explained below.

The attributes lack of practice and lack of application skills are inter connected . The attribute method of teaching is the cause for lack of practice, lack of application skills, low I.Q., short attention span. The attribute low I.Q. is the cause for lack of application skills, short attention span. The attribute short attention span is the cause for lack of practice and lack of application skills.

Using this Fig.2 and using the relations a related connection matrix $M_{2}$ is formed which is given below.

$$
M_{2}=\left[\begin{array}{lllll}
0 & 1 & 0 & 0 & 0 \\
1 & 0 & 0 & 0 & 0 \\
1 & 1 & 0 & 1 & 1 \\
0 & 1 & 0 & 0 & 1 \\
1 & 1 & 0 & 0 & 0
\end{array}\right]
$$

If there is relation between attributes we have given 1.Otherwise we have given 0 .

In Fig.3 using the attributes $G_{3}, G_{6}, G_{7}, G_{9}, G_{15}$ we have drawn a directed graph. We draw the graph based on the relation between the attributes $G_{3}, G_{6}, G_{7}, G_{9}, G_{15}$ which is explained below.

The attribute fear towards the subject is the cause for by hearting Mathematics. The attributes fear towards the subject and taking the course by force are interconnected. The attributes fear towards the subject and Attitude towards learning the subject are interconnected. The attributes taking the course by force and attitude towards learning the subject are interconnected.

Using this Fig.3 and using the relations a related connection matrix $M_{3}$ is formed which is given below.

$$
M_{3}=\left[\begin{array}{lllll}
0 & 0 & 0 & 0 & 0 \\
1 & 0 & 1 & 1 & 0 \\
0 & 1 & 0 & 1 & 0 \\
1 & 1 & 1 & 0 & 0 \\
0 & 0 & 0 & 0 & 0
\end{array}\right]
$$

If there is relation between attributes we have given 1.Otherwise we have given 0 . Now after getting the relational matrices $M_{1}, M_{2}, M_{3}$ we have combined the three matrices. Each matrix is referred as block. Combining the matrices is nothing but combining the blocks. So this technique is called Combined Disjoint Blocked Fuzzy Cognitive Maps. The combined matrix F is given below. 


\section{Reasons for the failure of B.Tech Students in Mathematics using Combined Disjoint Blocked Fuzzy Cognitive Maps (CDBFCM)}

$\mathrm{F}=\left[\begin{array}{lllllllllllllll}0 & 0 & 0 & 0 & 0 & 0 & 0 & 0 & 0 & 1 & 1 & 0 & 0 & 1 & 0 \\ 1 & 0 & 0 & 0 & 0 & 0 & 0 & 0 & 0 & 1 & 1 & 0 & 0 & 1 & 0 \\ 0 & 0 & 0 & 0 & 0 & 0 & 0 & 0 & 0 & 0 & 0 & 0 & 0 & 0 & 0 \\ 0 & 0 & 0 & 0 & 1 & 0 & 0 & 0 & 0 & 0 & 0 & 0 & 0 & 0 & 0 \\ 0 & 0 & 0 & 1 & 0 & 0 & 0 & 0 & 0 & 0 & 0 & 0 & 0 & 0 & 0 \\ 0 & 0 & 1 & 0 & 0 & 0 & 1 & 0 & 1 & 0 & 0 & 0 & 0 & 0 & 0 \\ 0 & 0 & 0 & 0 & 0 & 1 & 0 & 0 & 1 & 0 & 0 & 0 & 0 & 0 & 0 \\ 0 & 0 & 0 & 1 & 1 & 0 & 0 & 0 & 0 & 0 & 0 & 1 & 1 & 0 & 0 \\ 0 & 0 & 1 & 0 & 0 & 1 & 1 & 0 & 0 & 0 & 0 & 0 & 0 & 0 & 0 \\ 1 & 0 & 0 & 0 & 0 & 0 & 0 & 0 & 0 & 0 & 1 & 0 & 0 & 1 & 0 \\ 0 & 0 & 0 & 0 & 0 & 0 & 0 & 0 & 0 & 0 & 0 & 0 & 0 & 1 & 0 \\ 0 & 0 & 0 & 0 & 1 & 0 & 0 & 0 & 0 & 0 & 0 & 0 & 1 & 0 & 0 \\ 0 & 0 & 0 & 1 & 1 & 0 & 0 & 0 & 0 & 0 & 0 & 0 & 0 & 0 & 0 \\ 1 & 0 & 0 & 0 & 0 & 0 & 0 & 0 & 0 & 1 & 1 & 0 & 0 & 0 & 0 \\ 0 & 0 & 0 & 0 & 0 & 0 & 0 & 0 & 0 & 0 & 0 & 0 & 0 & 0 & 0\end{array}\right]$

For determining the hidden pattern we used the following method. Suppose $D_{1}, D_{2}, \ldots, D_{n}$ being nodes for the FCM having feedback. Suppose $\mathrm{W}$ is the connected adjacency matrix. We will determine the hidden pattern if $D_{1}$ be switched on. If we give entry as the vector $G_{1}$ $=(1,0, \ldots, 0)$, information must bypass all the way through the connection matrix $\mathrm{W}$. We can perform this by means of multiplying $G_{1}$ with the matrix W. Suppose $G_{1} \mathrm{~W}=\left(g_{1}, g, \ldots, g_{n}\right)$ having threshold function, it means that replicate $g_{i}$ with 1 when $g_{i} \geq \mathrm{k}$ , $g_{i}$ with 0 when $g_{i}<\mathrm{k}$ (k being appropriate positive integer). We reconstruct the resultant concept, the concept $D_{1}$ be incorporated into the reconstructed vector by means of taking the first coordinate be 1 inside the resultant vector. If $G_{1} \mathrm{~W}$ at this $G_{2} 7$ moment take $G_{2} \mathrm{~W}$, replicate the similar process. The process be replicated until we obtain a fixed point.

Now using this method the on position of the attribute lack of fundamentals was taken, each and every other states are off the effect of

$\mathrm{Y}=$ $\left(\begin{array}{lllllllllllllll}1 & 0 & 0 & 0 & 0 & 0 & 0 & 0 & 0 & 0 & 0 & 0 & 0 & 0 & 0\end{array}\right)$
on the CDBFCM is as follows.

YF

$\left(\begin{array}{lllllllllllllll}0 & 0 & 0 & 0 & 0 & 0 & 0 & 0 & 0 & 1 & 1 & 0 & 0 & 1 & 0\end{array}\right)_{\rceil}$ (Say) $Y_{1}=$

$Y_{1} \mathrm{~F}$

$\left(\begin{array}{lllllllllllllll}1 & 0 & 0 & 0 & 0 & 0 & 0 & 0 & 0 & 1 & 1 & 0 & 0 & 1 & 0\end{array}\right)_{7}$ (Say) $Y_{2}=$

$Y_{2} \mathrm{~F}$

$\left(\begin{array}{lllllllllllllll}1 & 0 & 0 & 0 & 0 & 0 & 0 & 0 & 0 & 1 & 1 & 0 & 0 & 1 & 0\end{array}\right)_{7}$ ${ }_{2}=\mathrm{Y} Y_{3}=$

$\mathrm{Y}_{2}$ will be the fixed point belonging to the dynamical system. If the state $G_{1}$ is on lack of fundamentals, lack of connection between the subject and students, self doubt and lack of understanding about signs and symbols are causes for failure.
If the on state of the attributes lack of fundamentals, lack of practice, taking the course by force, self doubt, remaining nodes are in off state.

At this moment we learn the effect of the dynamical system $\mathrm{F}$ Let

$$
=
$$

$$
\left(\begin{array}{lllllllllllllll}
1 & 0 & 0 & 1 & 0 & 0 & 1 & 0 & 0 & 0 & 1 & 0 & 0 & 0 & 0
\end{array}\right)
$$

be the state vector depicting the on state vector $\mathrm{T}$ inside dynamical system $\mathrm{F}$.

TF
7
$(0$

$$
\begin{aligned}
& \left(\begin{array}{lllllllllllllll}
0 & 0 & 0 & 0 & 1 & 1 & 0 & 0 & 1 & 1 & 1 & 0 & 0 & 1 & 0
\end{array}\right) \\
& \left(\text { Say) } T_{1}=\right. \\
& T_{1} \mathrm{~F} \\
& \left(\begin{array}{lllllllllllllll}
1 & 0 & 1 & 1 & 0 & 1 & 1 & 0 & 1 & 1 & 1 & 0 & 0 & 1 & 0
\end{array}\right)_{7} \\
& \left(\text { Say) } T_{2}=\right.
\end{aligned}
$$

\section{$T_{2} \mathrm{~F}$}

$\left(\begin{array}{lllllllllllllll}1 & 0 & 1 & 0 & 0 & 1 & 1 & 0 & 1 & 1 & 1 & 0 & 0 & 1 & 0\end{array}\right)_{\rceil}$ (Say) $T_{3}=$

$$
\begin{aligned}
& T_{3} \mathrm{~F} \\
& \left(\begin{array}{lllllllllllllll}
1 & 0 & 1 & 0 & 0 & 1 & 1 & 0 & 1 & 1 & 1 & 0 & 0 & 1 & 0
\end{array}\right)_{7}
\end{aligned}
$$$$
T_{3}={ }_{4} \mathrm{~T}=
$$

Hence $T_{3}$ will be the fixed point of the dynamical system We investigated the causes for the failure of B.Tech. Students in Mathematics with the help of CDBFCM model. The limit point of the dynamical system proves that the attributes $\mathrm{G}_{1}, \mathrm{G}_{3}, \mathrm{G}_{6}, \mathrm{G}_{7}, \mathrm{G}_{9}, \mathrm{G}_{10}, \mathrm{G}_{11}, \mathrm{G}_{14}$ are the most important causes for the failure of B.Tech. Students in Mathematics. This implies that lack of fundamentals, by hearting Mathematics, fear towards the subject, taking the course by force, attitude towards learning the subject, lack of connection between the subject and students, self doubt, lack of understanding about signs and symbols are the most important causes for the failure of B.Tech. Students in Mathematics and due to these causes the B.Tech. Students are getting failed in Mathematics.

\section{CONCLUSION}

Using CDBFCM Model we considered a social problem and identified the major causes. We have conducted manual survey from which we got the same result. Manual survey is time taken process. Instead of doing manual survey we can apply this model and we can do the work in an effective manner. So this technique is fast and effective.

\section{REFERENCES}

1. A. Victor Devadoss, M .Clement Joe Anand, "Dimensions of Personality of Women in Chennai Using CETD Matrix", InternationalJournal of Computer Applications, July-2012.

2. B. Kosko, "Fuzzy Cognitive Maps", International Journal of man-machine studies, January, (1988), 62-75.

3. B. Kosko, "Hidden patterns in combined and Adaptive Knowledge Networks", Proc. Of the First, IEE International Conference on Neural Networks (ICNN-86(1988) 377-393.

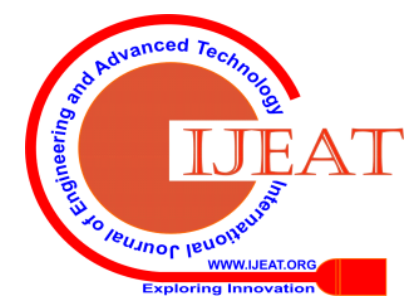


4. B. Kosko, "Neural Networks and Fuzzy systems: A Dynamical System Approach to Machine Intelligence", Prentice Hall of India, 1997.

5. George J. Klir / Bo Yuan, "Fuzzy sets and Fuzzy Logic: Theory and Applications", Prentice Hall of India.

6. H. J. Zimmermann, "Fuzzy Set Theory and its application", Fourth Edition Springer 2011.

7. Programme evaluatin report: Activity Based Learning Tamil Nadu, National council of educational research and training, December 2011.

8. R. Axelrod, "Structure of decision: The cognitive maps of political elites". Princeton, N.J: Princeton University Press, 1976.

9. W. B. Vasantha Kandasamy and A.Victor Devadoss, "Some New Fuzzy Techniques", Jour. of Inst. of. Math. \& Comp. Sci. (Math. Ser.), Vol. 17, No.2, (2004), 157- 160

10. G.Anusha, P. Venkataramana"A study on Symptoms of stress on college students using Combined Disjoint Block Fuzzy Cognitive Maps(CDBFCM)"in INTERNATIONAL JOURNAL OF ADVANCES IN APPLIED MATHEMATICS AND MECHANICS. ISSN-2347-2529,Vol-2(3)-March2015,177-182.

11. G. Anusha, N. Srinivasarao.” Effect of Playing violent on line Games on Students using Combined Disjoint Blocked Fuzzy Cognitive Maps (CDBFCM)"'in INTERNATIONAL JOURNAL OF INNOVATIVE TECHNOLOGY AND EXPLORING ENGINEERS.-ISSN-2278-3075, Vol-8(8)-June2019, 2002-2007.

\section{AUTHORS PROFILE}

G.Anusha. Qualification is M.Sc., M.Phil., (P.hd). Working as Assistant Professor in Vignans Lara Institute of Technology and Scinces. 8 research papers published in international journals. 